\title{
Fechamento de diastema pela técnica direta em resina composta: relato de caso clínico
}

\author{
Diastema closure by direct technique in composite resin - a case report \\ Cierre del diastema por técnica directa en resina compuesta - reporte de un caso
}

Laryssa Marques de Oliveira e Oliveira ORCID: https://orcid.org/0000-0002-0251-5505 Centro Universitário Fametro, Brasil E-mail: laryssa545@gmail.com

Rayssa Nunes Bezerra

ORCID: https://orcid.org/0000-0002-2614-9589 Centro Universitário Fametro, Brasil E-mail: raynunesbezerra@outlook.com

Fernanda Thomáz do Carmo

ORCID: https://orcid.org/0000-0002-8834-4769 Centro Universitário Fametro, Brasil

E-mail: fernandathomaz1@ @otmail.com

Gabriela de Figueiredo Meira

ORCID: https://orcid.org/0000-0002-8285-8769 Universidades Federal de Santa Maria, Brasil E-mail: gabrielameira1@ hotmail.com Juliana Lopes de Sá

ORCID: https://orcid.org/0000-0002-1927-2191 Centro Universitário Fametro, Brasil E-mail: juliana.lopes@fametro.edu.br

Luciana Onety Ramalho

ORCID: https://orcid.org/0000-0003-4100-860X Centro Universitário Fametro, Brasil E-mail: luciana_onety@hotmail.com

\begin{abstract}
Resumo
Objetivo: O objetivo desse trabalho é relatar um caso clínico de fechamento de diastemas através da técnica direta de resina composta guiado por enceramento diagnóstico. Metodologia: Partindo da permissão da paciente por meio do Termo de Consentimento Livre e Esclarecido em partilhar sua imagem para devido fim, o presente estudo trata de um relato de caso clínico de cunho analítico descritivo (Pereira et al., 2018). Relato de Caso: Paciente do gênero feminino, 21 anos de idade, parda, compareceu a clínica odontológica do CEUNI-Centro Universitário Fametro. Na anamnese se mostrava insatisfeita com a estética do sorriso. Durante o exame clínico, foi observada a presença de diastemas envolvendo a região dos incisivos centrais e laterais superiores. Considerações Finais: A reabilitação estética através da técnica direta em resina composta é uma excelente alternativa para o fechamento de diastemas, desde que realizados da maneira correta levando em consideração a habilidade do operador e a colaboração do paciente após o tratamento.
\end{abstract}

Palavras-chave: Faceta; Resina; Diastema.

\begin{abstract}
Objective: The objective of this work is to report a clinical case of diastema closure using the direct resin technique guided by diagnostic waxing. Methodology: Based on the patient's permission through the Free and Informed Consent Term to share their image for the proper purpose, this study is a clinical case report of descriptive analytical nature (Pereira et al., 2018). Case Report: Female patient, 21 years old, brown, attended the dental clinic of CEUNI-Centro Universitário Fametro. In the anamnesis, she showed dissatisfaction with the aesthetics of the smile. During clinical examination, the presence of diastemas involving the region of the upper central and lateral incisors was observed. Final Considerations: Aesthetic rehabilitation through the direct technique in composite resin is an excellent alternative for closing diastemas, as long as it is done correctly, taking into account the operator's skill and the patient's cooperation after the treatment.
\end{abstract}

Keywords: Facet; Resin; Diastema. 


\begin{abstract}
Resumen
Objetivo: El objetivo de este trabajo es reportar un caso clínico de cierre de diastema mediante la técnica de resina directa guiada por encerado diagnóstico. Metodología: Con base en el permiso del paciente a través del Término de Consentimiento Libre e Informado para compartir su imagen con el propósito adecuado, este estudio es un reporte de caso clínico de naturaleza analítica descriptiva (Pereira et al., 2018). Caso clínico: Paciente de sexo femenino, 21 años, morena, que acudió a la consulta odontológica del CEUNI-Centro Universitário Fametro. En la anamnesis, mostró insatisfacción con la estética de la sonrisa. Durante el examen clínico se observó la presencia de diastemas que afectaban la región de los incisivos centrales superiores y laterales. Consideraciones finales: La rehabilitación estética a través de la técnica directa en resina compuesta es una excelente alternativa para cerrar diastemas, siempre que se realice correctamente, teniendo en cuenta la habilidad del operador y la cooperación del paciente después del tratamiento.
\end{abstract}

Palabras clave: Faceta; Resina; Diastema.

\title{
1. Introdução
}

A odontologia estética está diariamente passando por avanços e a resina composta é um material cada vez mais presente dentro dos consultórios odontológicos, por apresentar uma possibilidade de tratamento minimamente invasivo e um custo relativamente baixo para o paciente (Campos et al., 2016). De acordo com Silva (2015), a aparência do sorriso tem levado pacientes a buscarem tratamentos estéticos para promover a harmonia do sorriso. Com a crescente procura para satisfazer as necessidades estéticas, dispõe-se da possibilidade do uso de resinas compostas.

Há uma gama de possibilidades com as resinas compostas, desde a reabilitação de um único elemento dentário, a remodelação de todo sorriso de um paciente, sendo possível trabalhar a mudança de cor e até mesmo a redefinição do formato dos elementos dentários. (Rodrigues et al., 2014). As restaurações diretas de resina composta, proporcionam um tratamento estético e funcional conservador por preservar o máximo de tecido dental possível. O sucesso clínico do procedimento depende do manuseio correto do material restaurador e da indicação adequada para o tratamento (Berwander et al., 2016).

Dentre as alterações que afetam a aparência do sorriso está o diastema, que são espaços interdentais que podem acometer toda a arcada dentária, causando maior incômodo quando se apresentam nos elementos ântero-superiores. Por se tratar de uma região estética, os pacientes buscam por uma alternativa para o fechamento dos mesmos (Guerra et al., 2017). Ferreira (2019) diz que a reanatomização dentária através da técnica direta de resina composta, é uma das alternativas parar o fechamento de diastemas, trazendo resultados estéticos e funcionais satisfatórios.

A estética do sorriso é um fator que influencia diretamente a autoestima de muitos indivíduos, e a desarmonia do sorriso causada pelos diastemas leva o paciente a procurar tratamentos que vão desde a confecção de laminados cerâmicos a técnica de restauração direita em resina composta. (Correa et al., 2020).

O valor elevado e o tempo de espera para conclusão de um tratamento com laminados cerâmicos, tem levado cirurgiões dentistas e pacientes a procurar uma alternativa com um menor custo, tempo de trabalho mais rápido, e desgaste dentário mínimo, tornando assim, a resina composta o material ideal para esse caso. (Shcwarz et al.,2013).

Destarte, esse trabalho tem o objetivo de trazer um relato de caso clínico de fechamento de diastemas pela técnica direta de resina composta em uma paciente descontente com a aparência do sorriso.

\section{Metodologia}

Partindo da permissão da paciente por meio do Termo de Consentimento Livre e Esclarecido em partilhar sua imagem para devido fim, o presente estudo trata de um relato de caso clínico de cunho analítico descritivo (Pereira et al., 2018) sobre fechamento de diastemas através da técnica direta de resina composta, realizado na clínica odontológica do CEUNI Fametro, na disciplina de estágio supervisionado em Clínica Integrada III, do curso de graduação em odontologia do Centro Universitário Fametro. 


\section{Resultados e Discussão}

Paciente do gênero feminino, 21 anos de idade, parda, compareceu a clínica odontológica do CEUNI-Centro Universitário Fametro. $\mathrm{Na}$ anamnese se mostrava insatisfeita com a estética do sorriso.

Durante o exame clínico, foi observada a presença de diastemas envolvendo a região dos incisivos centrais e laterais superiores (Figura 1).

Figura 1 - Aspecto inicial mostrando a presença de diastemas.

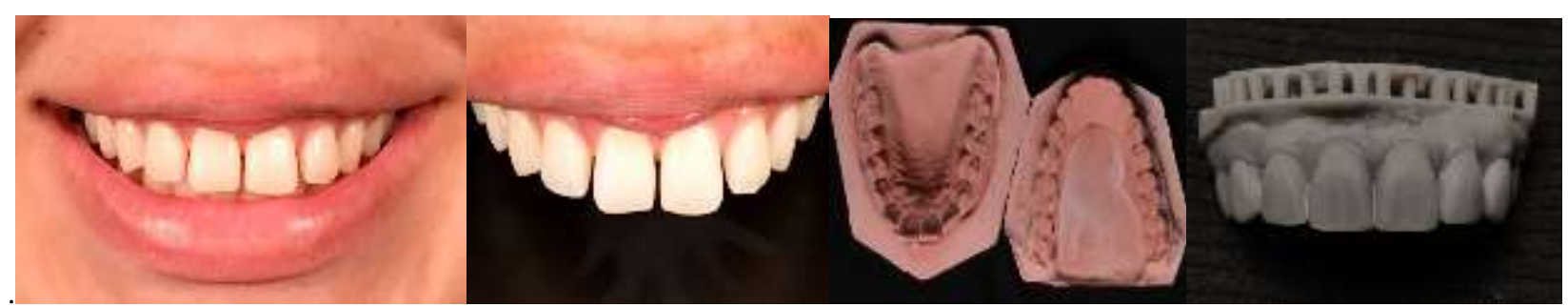

Fonte: Autores.

Inicialmente, foi realizada a moldagem com silicone de condensação (Perfil putty denso- vigodent coltene) da arcada superior e inferior e vazados em gesso especial tipo IV (Yamay Venus TP). Os modelos foram enviados ao laboratório para a realização do enceramento diagnóstico.

Inicialmente foi realizada profilaxia com pedra pomes. Em seguida foi feita a seleção de cor da resina composta através da técnica de pérolas, onde pequenos incrementos de resina foram adicionados na porção cervical, terço médio e incisal dos elementos dentários sem o uso de condicionamento ácido e sistema adesivo. Os incrementos de resina composta foram fotopolimerizados por 20 segundos e as cores selecionadas foram EA1 (Vittra APS-FGM) para a confecção da parede palatina e DA1 (Vittra APS- FGM) para face vestibular (Figura 2).

Figura 2 - A) Isolamento absoluto; B) Aplicação do ácido fosfórico a 37\%.

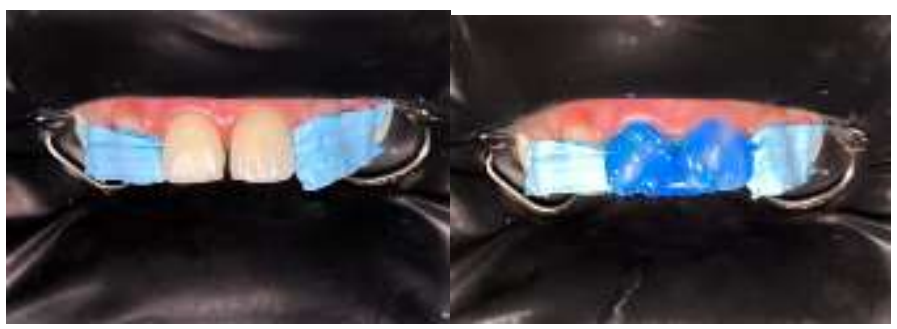

Fonte: Autores.

Foi realizado isolamento absoluto modificado com arco de young, grampos $\mathrm{N}^{\circ} 0$, pinça palmer e lençol de borracha. Com auxílio do Isotap (TDV) foi feito o isolamento dos dentes adjacentes (Figura 6) para a aplicação do ácido fosfórico a $37 \%$ durante 30 segundos na face vestibular, palatina e na região proximal dos elementos dentários.

Após 30 segundos foi feita retirada do ácido fosfórico com auxílio de rolete de algodão e lavagem abundante com água. Em seguida foi realizada a secagem e aplicação do adesivo (Ambar-FGM) e fotopolimerização por 30 segundos (Figura $3)$. 
Figura 3 - Aplicação do adesivo com auxílio de um aplicador micro brush.

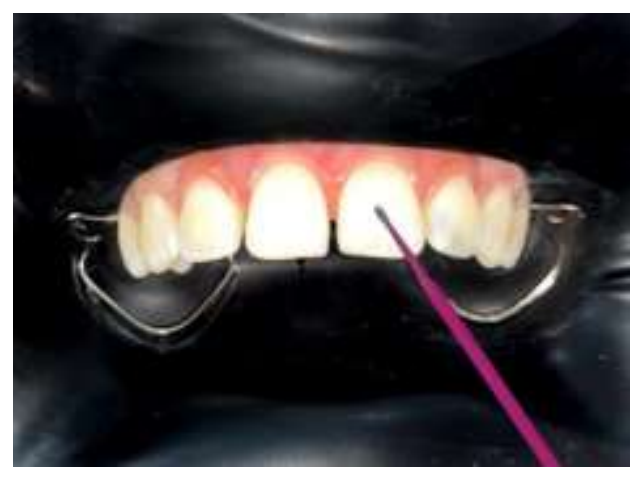

Fonte: Autores.

Com auxílio da guia de silicone obtida a partir do enceramento diagnóstico, foi confeccionada a parede palatina. Em seguida, foi realizada a inserção da resina composta na cor EA1 (Vittra APS-FGM) na guia de silicone que foi levada à cavidade e posicionada, onde foi fotopolimerizada durante 20 segundos (Figura 4).

Figura 4 - Posicionamento da guia palatina.

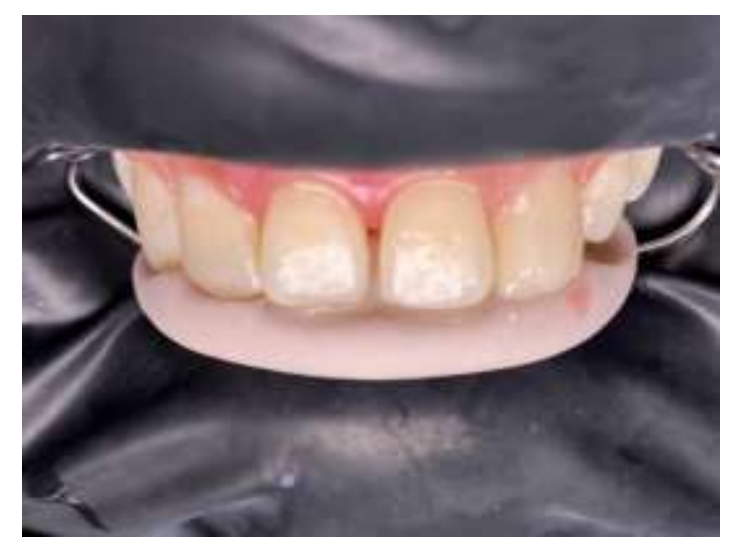

Fonte: Autores.

Após a polimerização, a guia de silicone foi removida, e a resina composta foi adicionada na face vestibular do dente em pequenos incrementos com auxílio da espátula de inserção número 1 e pincel de pelo sintético n²4 (Tokuyama).

Utilizou-se tira de poliéster (TDV) na região proximal dos elementos dentários, em seguida foi feita a remoção de excessos de material restaurador da região cervical com auxílio de brocas diamantadas FF e ajuste de oclusão.

Após sete dias, foi realizado o acabamento e polimento com brocas multilaminadas, discos de polimento de granulação de maior para menor (TDV), kit de acabamento e polimento (Ultra- gloss, American Burrs). discos de feltro (Diamond- FGM) e pasta de polimento (Diamond Pro- FGM) (Figura 5) 
Figura 5 - A) Polimento com disco de granulação; B) Polimento com Kit Ultra-Gloss; C) Polimento com disco de feltro; D) Aspecto Final.

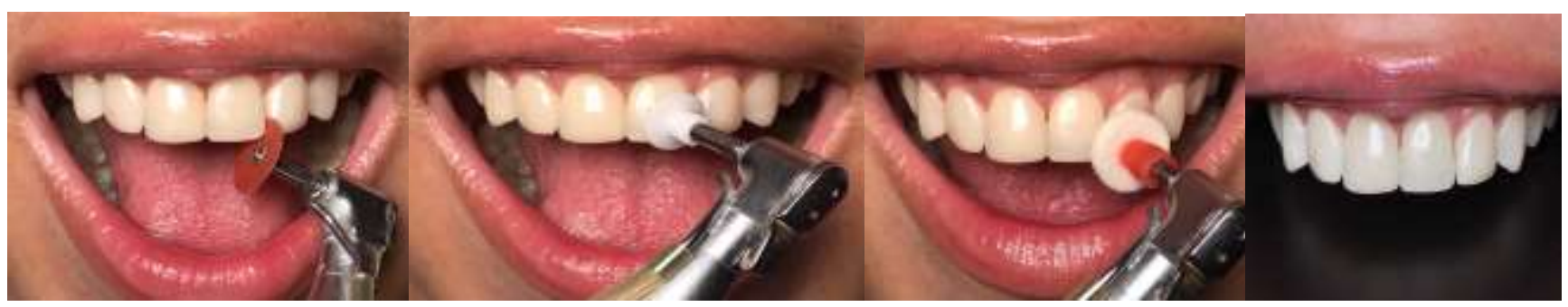

Fonte: Autores.

\section{Discussão}

A procura pelo sorriso perfeito tem levado pacientes a buscarem tratamentos estéticos para devolver a harmonia do sorriso (Correa et al., 2020). No presente caso, a paciente se mostrava insatisfeita com aparência dos dentes por conta da presença de diastemas, como tratamento proposto foram indicadas as facetas em resina composta.

Higashi et al., (2006) considera que a técnica direta de resina composta é uma solução satisfatória para o fechamento de diastemas já que as resinas oferecem resultados estéticos e funcionais, podendo devolver o contorno, volume e funcionalidade dos elementos dentários. Segundo Cardoso et al., (2011) a técnica direta de resina composta tem como vantagem a preservação de estrutura dentária, já que o desgaste dental é mínimo ou inexistente. Por dispensar etapas laboratoriais como as facetas indiretas, o tempo para confecção das facetas em resina composta é menor.

No caso apresentado foi confeccionado o enceramento diagnóstico, partindo da ideia de se realizar um procedimento conservador, onde é possível devolver a funcionalidade e estética do sorriso. De acordo com o trabalho de Goyatá et al., (2017) enceramento diagnóstico é uma forma de trazer uma maior previsibilidade da aparência estética final. Essa etapa torna possível que o paciente veja e aprove o tamanho e formato dos dentes antes da confecção das facetas.

Em 2001, Baratieri descreveu a técnica da matriz palatina obtida a partir do enceramento diagnóstico que é indicada para elementos dentários com alteração de cor, problemas de posição ou formato dos dentes, a fim de promover uma melhor estética e facilitar o ajuste oclusal. No referido caso, foi utilizada a técnica descrita por Baratieri que se dá através da moldagem prévia do enceramento diagnóstico com silicone de condensação. A partir dessa moldagem obteve-se a matriz palatina onde foi possível reproduzir a face palatina e a região incisal dos elementos dentários nas quais foram confeccionadas as facetas.

De acordo com Benevides (2019) o isolamento absoluto é indispensável para a realização da técnica direta de resina composta, pois se tem um maior controle de umidade e diminui o risco de contato com a saliva. Para uma melhor acomodação da guia palatina, optou-se pelo isolamento absoluto modificado.

Souza et al., (2002) disse que as restaurações diretas são pequenos compósitos de material restaurador adicionados gradualmente na estrutura dentária que recobrem a face vestibular do dente onde a adesão desse material no elemento dentário se dá a partir de um agente de união, o sistema adesivo. No caso descrito, foram confeccionadas seis facetas em resina composta, onde o material restaurador foi aplicado diretamente nos elementos dentários em pequenos incrementos.

Coimbra et al., (2016) considera que o sucesso estético e funcional das restaurações diretas em resina composta depende da habilidade do cirurgião-dentista, que deve ter conhecimento básico do material que utilizará para a confecção das facetas, como adesivos e resinas compostas. Vale ressaltar a importância do trabalho multidisciplinar, já que o sucesso do tratamento requer uma correta indicação. 
Para Campagnolo et al., (2019) um aspecto importante para as facetas em resina composta é o acabamento e polimento que são obtidos com brocas multilaminadas, discos de sequência de polimento de maior para menor granulação, taças de borracha, discos de feltro e pasta de polimento para promover a lisura superficial e devolver o aspecto natural dos elementos dentários, Entretanto, a aparência das facetas a longo prazo se dá a partir dos cuidados do paciente, sendo necessário manter uma boa higienização afim de evitar o manchamento e manter a lisura superficial da faceta. (Bernardes, 2017). No caso descrito, foi realizado o acabamento e polimento na última sessão, onde foi possível devolver o brilho e a naturalidade dos dentes.

Para Souza et al., (2002) as desvantagens da técnica direta contraposto a técnica indireta são de instabilidade de cor, já que a resina composta tem maior propensão a manchamento e menor resistência em relação aos laminados cerâmicos, em contra partida, os laminados cerâmicos exigem um maior desgaste de estrutura dentaria, maior tempo para conclusão do tratamento e tem um maior custo. Nesse presente caso, não se fez necessário o desgaste de nenhum dente, preservando a saúde do elemento dentário e tornado o procedimento conservador.

É de extrema importância a elaboração de um plano de tratamento e a correta indicação da técnica direta em resina composta para o fechamento de diastemas. Sendo possível entregar um sorriso perfeito, pode-se utilizar as facetas em resina composta, como descrito neste relato de caso.

\section{Considerações Finais}

A reabilitação estética através da técnica direta em resina composta é uma excelente alternativa para o fechamento de diastemas, desde que realizados da maneira correta levando em consideração a habilidade do operador e a colaboração do paciente após o tratamento. O uso do enceramento diagnóstico foi primordial para se obter uma maior previsibilidade do tratamento juntamente com a guia palatina, que possibilitou a confecção das facetas de forma harmônica. Como descrito no caso presente, obteve-se um ótimo resultado estético, devolvendo a harmonia do sorriso e atendendo as expectativas estéticas da paciente.

\section{Referências}

Benevides, A. A., Venâncio, A. E. F., \& Feitosa, V. P. (2019). A influência do isolamento absoluto no sucesso de restaurações diretas e tratamento endodôntico: uma revisão de literatura. Rev. Odontol. de Araçatuba (Impr.), 40(1), 35-40.

Bernardes, A. C. T. A. A. (2017). Seleção de cor para a obtenção de restaurações anteriores diretas imperceptíveis. Relatório Final de Estágio, Instituto Universitário de Ciências da Saúde, Gandra, Portugal.

Berwanger C. et al. (2016). Fechamento de diastema com resina composta- relato de caso clínico. Rev. Assoc. Paul. Cir. Dent. 20(3). São paulo- ISSN- 00045276.

Campagnolo, V., Antunes, E. L., Cemin, J. F., Pissaia, J. F., Pissaia, J. F., \& Campos, L. A. (2019). Correção de diastemas por meio de restaurações diretas em resina composta: relato de caso clínico.

Cardoso, P. C., Decurcio R. A., Pacheco, A. F. R., Monteiro, L. J. E., Ferreira, M. G., Lima, P. L. A., Silva, R. F. K. (2011). Facetas diretas de resina composta e clareamento dental:estratégiaspara dentes escurecidos, RevOdontolBras Central. 20(55):341-7.

Coimbra Júnior, N. Da C., Guerino, P., Mezomo, M. B. (2016). Diastemas interincisais superiores revisão acerca da etiologia, tratamento e estabilidade em longo prazo. Disciplinarum Scientia, 17(1), 95-109.

Silva, G. R. et al. (2016). Tratamento estético com diretas de resina composta- relato de caso. Revista UNINGÁ Review, Maringá 24(3), 27-3- ISSN 2178 2571.

Reis Goyatá, F., Moreno, A., Lanza, C. R. M., Barreiros, I. D., Novaes Jr, J. B., \& Goyatá, L. F. R. (2018). Restauração dos dentes anteriores superiores com resina composta.

Ferreira, F. S. et al. Fechamento de diastema com resina composta. Anais da jornada odontológica de Anápolis. P. 187-190 - ISSN 2596-116.

Guerra, M. L. R. S., et al. (2017). Fechamento de diiastemas anteriores com resina composta direta: relato de caso. FOL- Faculdade de Odontologia Lins/ Unimep, 27(1), 63-68. ISSN-2238-1236. 
Research, Society and Development, v. 10, n. 16, e431101624060, 2021 (CC BY 4.0) | ISSN 2525-3409 | DOI: http://dx.doi.org/10.33448/rsd-v10i16.24060

Hafez, R., Ahmed, D., Yousry, M., El-Badrawy, W., \& El-Mowafy, O. (2010). Effect of in-office bleaching on color and surface roughness of composite restoratives. Eur J Dent., 4, 118-27.

Higashi, C., Gomes, J. C., Kina, S., Andrade, O. S., \& Hirata, R. (2006). Planejamento estético em dentes anteriores. Odontologia Estética 7, 139-54.

Kabbach, W. (2018). Diastema closures: A novel technique to ensure dental proportion. Journal of Esthetic and Restorative Dentistry, 30(4), 275-280.

Lima, H. E., R. et al. (2020). Fechamento de diastema utilizando resina composta. Braz. J. of Develop., Curitiba, 6(12), 95036-95045, ISSN 2525-8761.

Monte-Alto et al., (2011). Tratamento estético restaurador com uso de resina composta em dentes anteriores associado a clareamento dentário prévio: relato de caso clínico. Revista Dental Press de Estética. 8 Issue 2, 66-76, 25.

Okida, R. C., et al., (2011). Emprego de técnica cirúrgica e materiais adesivos diretos no fechamento de diastemas. RPG, Rev. pós-grad, 18(1),57-61. ISSN 0104-5695.

Pereira, M.R., et al. (2020). Reabilitação estética com resina composta em paciente jovem: Relato de caso clínico. Rev Odontol Bras P. $20-28$ v29i88.1296.

Pereira, A. S., Shitsuka, D. M., Parreira, F. J., \& Shitsuka, R. (2018). Metodologia da pesquisa científica. Santa Maria, RS: UFSM, NTE.

Rodrigue S. D. R. et al. (2014). Reanatomização dental com resina composta - Relato de Caso. Revista Bahiana de Odontologia, 5(3),182-192.

Shcwarz, V. et al. Fechamento de diastema com resina composta: relato de caso. J Oral Invest, 2(1): 26-31, 2013 - ISSN 2238-510X.

Souza, E. M., Silva e Souza J. R. M. H., Lopes, F. A. M., \& Oster- nack, F. H. R. (2002). Facetas estéticas indiretas em porcelana, JBD, 1(3):256-62 